\title{
Principales causas de ausentismo laboral: una aplicación desde la minería de datos
}

\author{
Emmanuel A. Berón, Daniela Mejía y Omar D. Castrillón \\ Universidad Nacional de Colombia, Facultad de Ingeniería y Arquitectura, Departamento de Ingeniería Industrial, \\ Campus La Nubia Bloque Q piso 2, Manizales - Colombia. (correo-e: eaberonm@unal.edu.co, dmejiap@unal.edu.co, \\ odcastrillong@unal.edu.co)
}

Recibido Jul. 24, 2020; Aceptado Sep. 24, 2020; Versión final Oct. 20, 2020, Publicado Abr. 2021

\begin{abstract}
Resumen
El objetivo fundamental de este trabajo es predecir el comportamiento de "motivo ausentismo" (variable dependiente) mediante técnicas de minería de datos. Se estudian diez variables independientes: sexo, edad, contrato, hijos, casado, antigüedad, turno, trabajo, sindicalizado y escolaridad. La variable dependiente se examina en dos estados: enfermedad y calamidad. Por medio del algoritmo J48 (Weka), se selección las variables independientes más influyentes y se predice el comportamiento de la variable dependiente. Los resultado muestran, con efectividad superior al $94.72 \%$, que las variables independientes más influyentes en el ausentismo laboral son: sindicalizado, hijos, sexo, contrato, estudios, casado y antigüedad. Las otras variables (salario, turno, y edad) parecen tener menos influencia. Se concluye que el desarrollo metodológico llevado acabo define una forma objetiva para resolver un problema tradicionalmente analizado de forma subjetiva y que puede ser replicable si se siguen los pasos estructurados en la metodología propuesta.
\end{abstract}

Palabras clave: minería de datos; ausentismo laboral; correlación; influencia moderada

\section{Main causes of workplace absenteeism: applying data mining}

\begin{abstract}
The main objective of this research study is to predict the behavior of motives (dependent variable) that cause workplace absenteeism by applying data mining techniques. Ten independent variables are examined: gender, age, job contract, having children, marriage status, labor seniority, schedule, work, unionized, and education. The dependent variable is examined in two states: disease and calamity. The J48 algorithm (Weka) is used to select the most influential independent variables and the behavior of the dependent variable. The results show that, with over $94.72 \%$ accuracy, the most influential independent variables on workplace absenteeism are: unionized, having children, gender, marriage status, labor seniority. The other variables (salary, hours, and age) examined appeared to have less influence. It is concluded that the methods developed here provide an objective way to resolve a problem that is traditionally examined subjectively and that the method can be replicated if the methodology proposed is followed as step-by-step.
\end{abstract}

Keywords: data mining; work absenteeism; correlation; moderate influence 


\section{INTRODUCCIÓN}

La Organización mundial de la salud (OMS), define el ausentismo como: la inasistencia por causas justificadas o injustificadas al trabajo de un empleado. Igualmente, se considera que existe ausentismo laboral, cuando el empleado asiste al trabajo, pero realiza actividades no relacionadas con el mismo. En este mismo sentido, la Organización Internacional del trabajo (OIT) define el ausentismo como la inasistencia al trabajo de alguien que debía hacerlo (Cuevas et al., 2011). Aspectos que generan grandes sobre costos y desordenes empresariales, con las subsecuentes perdidas. Siendo quizás esta una de las razones que justifiquen la existencia de diversos estudios en esta área. Una primera revisión literaria muestra que este problema ha sido analizado desde dos puntos de vista diferentes. El primero de ellos se basa en un análisis físico, psicológico y social de las personas, mientras que el segundo punto de vista analiza este problema desde las diferentes técnicas de inteligencia artificial. En el primer punto de vista se encuentran diversos autores (Restrepo y Salgado, 2013), quienes, en condiciones de trabajo inciertas y tomando como referencia encuestas preestablecidas han encontrado que los trabajadores con contratos formales y las mujeres (en mayor porcentaje casadas) tienen una probabilidad más alta de faltar al trabajo, en contraste con las personas que presentan un nivel educativo más bajo o que pertenecen a empresas más pequeñas.

Igualmente, investigaciones desarrolladas por otros autores (Heinemann et al., 2010) analizan los síntomas premenstruales leves o moderados en mujeres entre 15 y 45 años, previos al ausentismo en el trabajo y su productividad, tomando como base el desarrollo de un cuestionario prestablecido. Otros autores (Halbesleben et al., 2014) analizan la decisión de asistir al trabajo aun cuando el trabajador está enfermo, (presentismo), el cual puede tener costos laborales y productivos aún mayores que los del ausentismo. Este hecho junto con el ausentismo aparentemente son dos caras de una misma moneda. No obstante, los autores encuentran que ambos hechos tienen orígenes diferentes, basados en las relaciones cambiantes, generadas en el tiempo, entre empleados y supervisores. En este mismo sentido otros estudios (Strömberg et al., 2017) estiman el impacto económico del presentimo y el ausentismo en la productividad, en un estudio basado en encuestas realizadas por gerentes de diversos sectores. Como resultado se encuentra una relación entre el trabajo, la pérdida de productividad, y el ambiente salud - trabajo.

Algunos otros autores (Bennedsen et al., 2019), han estudiado los diferentes factores del ausentismo, clasificándolos en factores empresariales (incentivos, cultura) y personales (motivación, ética), como resultado de este estudio, estos autores encontraron que los factores empresariales explican hasta en un $60 \%$ las diferencias de ausentismo entre las distintas empresas. En estos estudios el ausentismo en empresas familiares con mayores incentivos parece disminuir. Enfoques similares han sido propuestos por otros autores (Ben-David et al., 2017), donde se diseñan estrategias de ventilación y consideración de compensaciones con el fin de reducir el ausentismo y aumentar el rendimiento productivo. En un sentido similar (Askun-Celik y Unler-Oz, 2001), un estudio realizado en empleados de un centro de atención telefónica, se encuentra que las percepciones sobre la calidad de vida son factores influyentes en el ausentismo de las personas y sus rotaciones. Igualmente, otros trabajos (Cucchiella et al., 2014) analizan la gestión del ausentismo en el lugar de trabajo, con el fin de tener una visión más amplia sobre este problema y poder establecer posibles soluciones.

Así mismo, es importante resaltar que el ausentismo en el trabajo también puede ser ocasionado por otra serie de enfermedades ó factores relacionados como: depresión, dolor, fatiga, ritmo, postura, horas de trabajo, distancia al lugar de trabajo, duración del descanso, etc (Saidane et al., 2018). Igualmente, el ausentismo o presentimos puede estar influenciado por otra serie de enfermedades como artritis (Verstappen, S., 2015), en este estudio se encuentran ausentismos o presentimos en pacientes con esta patología, lo cual puede llevar a la empresa a costos muy altos, superiores a los costos directos. Así mismo, algunos estudios demuestran que incluso las vacunas pueden tener un impacto significativo en los ausentismos (Murti et al., 2019a; Murti et al., 2019b). Por su parte otras investigaciones (Johnston et al., 2019) muestra la relación lineal negativa existente entre la gravedad de la depresión y el presentimos, al mismo tiempo que muestran una relación estadísticamente significativa entre la gravedad de la depresión y el ausentismo. Estudios similares pueden ser encontrados en los siguientes artículos (Enns et al., 2015; Hernández-Martínez et al., 2020; Karatepe et al, 2020).

Desde el segundo punto de vista, algunos autores (Thales y Costa, 2020), han estudiado el problema del ausentismos desde el área de la inteligencia artificial. Estos autores, han empleado máquinas de aprendizaje automático para la predicción del ausentismo. Dichos modelos se basan en la identificación de las principales características de este problema. El modelo, es aplicado en una base de datos de las instituciones de seguridad pública, donde por medio de máquinas de soporte vectorial y redes neuronales se logra una efectividad del 78\%. Por su parte otros investigadores (Darvishi et al., 2017) emplean modelos basados en redes neuronales para predecir ciertas enfermedades como el dolor lumbar, las cuales pueden llevar aun ausentismo laboral. Este proceso logra una efectividad entre el $88 \%$ y $96 \%$, por medio de un modelo basado 
en 16 factores de riesgo, tomados de las interacciones ocupacionales, personales y psicológicos de una población de trabajadores. En un contexto más amplio, otras técnicas de inteligencia artificial, como la robótica, también han sido usadas para disminuir el tiempo de ausentismo producido por algunos procedimientos médicos (Pucheril et al., 2019).

Como se deduce de las diferentes revisiones literarias ilustradas en este trabajo, el ausentismo representa un aspecto bastante negativo para las organizaciones, el mismo puede acarrear grandes sobre cotos en los sistemas productivos constituyéndose en un problema de gran importancia que debe ser tratado. Se estima que, durante el 2018 en Colombia, se presentaron 1.59 casos de ausentismo laboral por cada trabajador con costos laborales que ascendieron a los $\$ 535.000 .000$ de dólares y costos médicos superiores a $\$ 177.000 .000$ millones de dólares (https://www.latinpymes.com. Así mismo y como se nota en las referencias de este documento, existen muy pocos análisis de este problema, en especial desde el campo de la inteligencia artificial, una razón más que justifica el desarrollo de esta investigación. Por ende, el objetivo fundamental de este trabajo es predecir, mediante técnicas de minería de datos, a partir de una serie de variables independientes (sexo, edad, contratos, hijos, casado, antigüedad, turno, trabajo, sindicalizado, estudios), el comportamiento de una variable dependiente denominada motivo ausentismo, con dos estados enfermedad o calamidad.

\section{MATERIALES Y METODOS}

El desarrollo de la metodología fue estructurado mediante el algoritmo de clasificación J48 (modificación del algoritmo C4.5 empleado en la construcción de árboles de decisión), desde la plataforma libre de aprendizaje automático y minería de datos Weka. Esta plataforma es descrita en (Witten et al., 2017). Esta metodología, estructura un archivo basado en las variables independientes analizadas (sexo, edad, contratos, hijos, casado, antigüedad, turno, trabajo, sindicalizado, escolaridad) y la variable dependiente que se desea predecir denominada motivo (ausentismo), con dos estados enfermedad o calamidad. Aspecto que permite estructurar los siguientes pasos: 1) Generación de la base de datos 2) estructuración del archivo para la plataforma Weka 3) Clasificación por medio del algoritmo J48 4) Identificación de las principales causas del ausentismo. Es importante resaltar que, el clasificador $\mathrm{J} 48$ empleado, permite obtener muy buenos resultados en comparación con otros clasificadores (Valencia et al., 2015; Castrillón et al., 2020; Castrillón et al., 2017).

\section{Paso 1. Generación de la base de datos}

Para la construcción de la base de datos, se tomó como referencia los registros del Departamento de Recursos Humanos de una empresa de la región central de Colombia. En total se tomaron 417 registros, los cuales contenían las siguientes campos o variables independientes: sexo (m, f), edad (18-30, 31-50, 51 o más), contrato (fijo, indefinido), hijos (si, no), casado (si, no), antigüedad (0-5, 5-10, 10 o más), turnos (diurno, nocturno), salario (1 a 2 mínimos, 3 a 4 mínimos, 5 o más mínimos) sindicalizado (si, no) escolaridad (bachiller, tecnológico, profesional). Igualmente se toma para cada registro la variable dependiente seleccionada.

\section{Paso 2. Estructuración del archivo para la plataforma Weka}

Las variables seleccionadas en el paso anterior, permiten estructurar el encabezado y el detalle del respectivo archivo .arff para ser interpretado desde la plataforma de aprendizaje automático y minería de datos denominada Weka, con el fin de obtener la clasificación correspondiente.

\section{Paso 3. Clasificación por medio del algoritmo J48}

El archivo .arff definido en el paso anterior, es interpretado por medio algoritmo de clasificación J48, desde la plataforma de aprendizaje automático y minería de datos Weka. La interpretación de este archivo, permitirá generar el respectivo árbol de clasificación. En este árbol los nodos representarán las causas (variables independientes) y las hojas contienen cada uno de los dos estados de la variable dependiente: enfermedad o calamidad. Además, la ejecución de este algoritmo permite obtener: a) La matriz de efectividad la cual ilustra el porcentaje de aciertos y errores en la clasificación. b) La matriz de precisión la cual muestra el porcentaje de positivos - verdaderos y falsos positivos c) La matriz de confusión representa los aciertos según los estados de la variable dependiente. d) El árbol de clasificación el cual ilustra la relación de las variables independientes conducentes a un estado especifico de la variable dependiente.

Paso 4. Identificación de las principales causas de ausentismo.

Con el fin de identificar las principales causas (variables independientes) que influyen sobre la variable dependiente, se realiza un recorrido longitudinal del árbol, desde la raíz hasta las cada una de las hojas. Este recorrido permite establecer la respectiva relación de causas y efectos. 


\section{RESULTADOS}

Como resultado de aplicar la metodología propuesta en cada uno de los pasos descritos en la sección anterior de este artículo se encuentra lo que sigue:

\section{Paso 1. Generación de la base de datos}

Cuando el Paso 1 de la metodología es aplicado, el siguiente archivo de base de datos, es definido. La Tabla 1 , muestra la estructura de este archivo.

Tabla 1: Base de datos de ausentismo. Por razones de espacio no se muestra el archivo completo

\begin{tabular}{|c|c|c|c|c|c|c|c|c|c|c|}
\hline $\begin{array}{l}\text { Sexo } \\
(F=114, \\
M=303)\end{array}$ & $\begin{array}{l}\text { Edad } \\
18 a 30=243, \\
31 a 50=103, \\
50+=71\end{array}$ & $\begin{array}{l}\text { Contrato } \\
\text { Fijo=40; } \\
\text { Indefinido } \\
=377\end{array}$ & $\begin{array}{l}\text { Hijos } \\
\text { Si = } \\
262, \\
\text { No }= \\
155\end{array}$ & $\begin{array}{l}\text { Casado } \\
\mathrm{Si}=225 \\
\mathrm{No}=192\end{array}$ & $\begin{array}{l}\text { Antigüedad } \\
0 \mathrm{a} 5=201, \\
5 \mathrm{a} 10=62, \\
10+=154\end{array}$ & $\begin{array}{l}\text { Turno } \\
\text { Diurno=2 } \\
06, \\
\text { nocturno } \\
=211\end{array}$ & $\begin{array}{l}\text { Salario } \\
\text { 1a2min=2 } \\
09, \\
\text { 3a4min=1 } \\
43,5+=65\end{array}$ & $\begin{array}{l}\text { Sind } \\
\text { Si= } \\
166, \\
\text { No=3 } \\
51\end{array}$ & $\begin{array}{l}\text { Escolaridad } \\
\text { bachiller=157, } \\
\text { tecnolo=58, } \\
\text { profesional } \\
202\end{array}$ & $\begin{array}{l}\text { Motivo } \\
\text { Enfer=278, } \\
\text { calamidad= } \\
139\end{array}$ \\
\hline Masculin & $18 \mathrm{a} 30$ & indefin & si & si & $0 \mathrm{a} 5$ & diurno & $1 \mathrm{a} 2$ & no & Prof & calamidad \\
\hline Masculin & $18 a 30$ & Indefin & si & si & $0 a 5$ & diurno & 1a2 & no & Prof & calamidad \\
\hline Masculin & $18 \mathrm{a} 30$ & Indefin & si & si & $0 \mathrm{a} 5$ & diurno & $3 a 4$ & no & Prof & calamidad \\
\hline . & & & . & & &. & &. &. &. \\
\hline masculin & 51omas & indefin & si & si & 10omas & diurno & 1a2 & si & bach & calamidad \\
\hline
\end{tabular}

\section{Paso 2. Estructuración del archivo para la plataforma Weka}

Con las variables empleadas, en el paso anterior, para construir la base de datos, se estructura el archivo para ser interpretado desde la plataforma de aprendizaje automático y minería de datos denominada Weka. Este archivo este estructurado en dos partes, como se ilustra en las Tablas 2 y 3 . El archivo es analizado por medio de un proceso de validación cruzada empleando el $80 \%$ de la base de datos para entrenamiento y $20 \%$ para la validación.

Tabla 2: Encabezado archivo .arff

\begin{tabular}{|l|l|l|}
\hline @RELATION & relation & \\
\hline @ATTRIBUTE & Sexo & $\{$ "femenino","masculino"\} \\
\hline @ATTRIBUTE & Edad & $\{$ "18a30","31a50","51omas" \\
\hline @ATTRIBUTE & Contrato & $\{$ "fijo", "indefinido"\} \\
\hline @ATTRIBUTE & Hijos & $\{$ "si", "no"\} \\
\hline @ATTRIBUTE & Casado & $\{$ "si", "no"\} \\
\hline @ATTRIBUTE & Antigüedad & $\{$ "0a5","5a10","10omas" $\}$ \\
\hline @ATTRIBUTE & Turno & $\{$ "diurno","nocturno" $\}$ \\
\hline @ATTRIBUTE & Salario & $\{$ "1a2minimos","3a4minimos","5omasminimos" $\}$ \\
\hline @ATTRIBUTE & Sindicalizado & $\{$ "si", "no"\} \\
\hline @ATTRIBUTE & Escolaridad & $\{$ "bachiller","tecnologo","profesional" $\}$ \\
\hline @ATTRIBUTE & Motivo & $\{$ "enfermedad","calamidad" $\}$ \\
\hline
\end{tabular}

Tabla 3: Detalle archivo .arff

\begin{tabular}{|l|l|l|l|l|l|l|l|l|l|l|}
\hline Sexo & Edad & Contrato & Hijos & Casado & Antigüedad & Turno & Salario & Sind & Escolaridad & Motivo \\
\hline Masculin & $18 \mathrm{a} 30$ & indefin & $\mathrm{si}$ & $\mathrm{si}$ & $0 \mathrm{a} 5$ & diurno & $1 \mathrm{a} 2$ & no & Prof & calamidad \\
\hline Masculin & $18 \mathrm{a} 30$ & Indefin & $\mathrm{si}$ & $\mathrm{si}$ & $0 \mathrm{a} 5$ & diurno & $1 \mathrm{a} 2$ & no & Prof & calamidad \\
\hline Masculin & $18 \mathrm{a} 30$ & Indefin & $\mathrm{si}$ & $\mathrm{si}$ & $0 \mathrm{a} 5$ & diurno & $3 \mathrm{a} 4$ & no & Prof & calamidad \\
\hline. &. &. &. &. &. &. &. &. &. &. \\
\hline masculin & 51 omas & indefin & $\mathrm{si}$ & $\mathrm{si}$ & 10omas & diurno & 1a2 & si & bach & calamidad \\
\hline
\end{tabular}

\section{Paso 3. Clasificación por medio del algoritmo J48}

Como resultado de realizar una clasificación del archivo .arff definido en las tablas 2 y 3 , por medio del algoritmo de clasificación J48, las respectivas matrices de efectividad, precisión y confusión, así como el árbol de clasificación son obtenidos, como se ilustra en las Tabla 4, 5, 6 y en la Figura 1, respectivamente. Así mismo, la Tabla 7 muestra la efectividad de esta metodología frente a otras técnicas de clasificación. Estas técnicas pueden ser encontradas en https://waikato.github.io/weka-wiki/. Igualmente, como se expresó en el punto anterior, se realiza una clasificación mediante un criterio de validación cruzada $80 \%$ y $20 \%$. Se resalta que analizando otras opciones de la base de datos con criterios de partición diferentes como $90 \%$ y $10 \%$ o $70 \%$ y $30 \%$ los resultados obtenidos son prácticamente iguales, razón por la cual se deja un valor de partición estándar, usualmente referenciado en la literatura. 
Tabla 4: Efectividad validación cruzada.

\begin{tabular}{|l|l|l|}
\hline Variable & Número & Porcentaje \\
\hline Correctly Classified Instances & 395 & 94.7242 \\
\hline Incorrectly Classified Instances & 22 & 5.2758 \\
\hline Kappa statistic & 0.8821 & \\
\hline Mean absolute error & 0.0835 & \\
\hline Root mean squared error & 0.2139 & \\
\hline Relative absolute error & $18.7802 \%$ & \\
\hline Root relative squared error & 45.3767 & \\
\hline Total Number of Instances & 417 & \\
\hline
\end{tabular}

Tabla 5: Matriz de precisión.

\begin{tabular}{|l|l|l|l|l|l|l|}
\hline Verdaderos + & Falsos + & Precisión & Recalculo & Medida- F & Area ROC & Clase \\
\hline 0.953 & 0.065 & 0.967 & 0.953 & 0.96 & 0.978 & Enfermedad \\
\hline 0.935 & 0.047 & 0.909 & 0.935 & 0.922 & 0.978 & Calamidad \\
\hline 0.947 & 0.059 & 0.948 & 0.947 & 0.947 & 0.978 & Promedio \\
\hline
\end{tabular}

Tabla 6: Matriz de confusión

\begin{tabular}{|c|c|c|}
\hline $\mathrm{a}$ & $\mathrm{b}$ & $\leftarrow$ Clasificación \\
\hline 265 & 13 & $\mathrm{a}=$ enfermedad \\
\hline 9 & 130 & $\mathrm{~b}=$ calamidad \\
\hline
\end{tabular}

Tabla 7: Efectividad del algoritmo J48 en compración con otras técnicas de clasificación

\begin{tabular}{|l|c|l|}
\hline Técnica & \%Aciertos & Descripción \\
\hline Adtree & 92.82 & Arbol de decisión alterno \\
\hline BFTree & 92.50 & Mejor primera decisión \\
\hline DesicioStump & 70.70 & Arbol de decisión de un nivel. \\
\hline Ft & 92.50 & Arboles funcionales \\
\hline Id3 & 92.50 & Árbol de decisión sin podar \\
\hline J48graft & 93.20 & árbol de decisión \\
\hline LADtree & 92.30 & Árbol de deción alterno de multiples clases \\
\hline LMT & 92.82 & Árbol de modelo logístico \\
\hline NBTree & 93.20 & Clasificador Naives Bayes \\
\hline RandomForest & 92.50 & Bosques de árboles aleatorios \\
\hline Randomtree & 92.50 & Árbol con k atributros elegidos de forma alazar en cada nodo \\
\hline Reptree & 93.20 & Árbol de aprendizaje de deción rapida \\
\hline Simpcart & 93.20 & Árbol de poda con el costo de complejidad minimo \\
\hline
\end{tabular}

\section{Paso 4. Identificación de las principales causas de ausentismo}

Tomando como referencia los resultados ilustrados en el paso 3, se encuentra que con una efectividad superior al $94.72 \%$ las variables más influyentes en un ausentismo laboral son en orden de importancia: Sindicalizado, hijos, sexo, contrato, escolaridad, casado, antigüedad, salario, turno y edad. Este aspecto es ilustrado en la Tabla 8.

Tabla 8: Identificación de causas de ausentismo

\begin{tabular}{|l|l|l|l|l|l|l|}
\hline \multicolumn{1}{|c|}{ Nodo 1} & Nodo/Hoja & \multicolumn{1}{|c|}{ Nodo/Hoja } & \multicolumn{1}{|c|}{ Nodo/Hoja } & Nodo/Hoja & Nodo/Hoja & Nodo/Hoja \\
\hline Sindicali (si) & Calamidad & & & & & \\
\hline Sindicali (no) & Hijos (si) & Sexo (femenino) & Calamidad & & & \\
\hline Sindicali (no) & Hijos (si) & Sexo (masc) & Escolar (bachi) & Antig (0a5) & Enfermedad & \\
\hline Sindicali (no) & Hijos (si) & Sexo (masc) & Escolar (bach) & Antig (5a10) & Calamidad & \\
\hline Sindicali (no) & Hijos (si) & Sexo (mascu) & Escolar (bach) & Antig (10+) & Enfermedad & \\
\hline Sindicali (no) & Hijos (si) & Sexo (mascu) & Escolar (tecnó) & Enfermedad & & \\
\hline Sindicali (no) & Hijos (si) & Sexo (mascu) & Escolar (Prof) & Sala (1a2) & Calamidad & \\
\hline Sindicali (no) & Hijos (si) & Sexo (mascu) & Escolar (Prof) & Sala (3a4) & Turno (noct) & Calami \\
\hline Sindicali (no) & Hijos (si) & Sexo (mascu) & Escolar (Prof) & Sala (3a4) & Turno (diur) & Enferm \\
\hline Sindicali (no) & Hijos (si) & Sexo (mascu) & Escolar (Prof) & Sala (5+) & Edad (18a30) & Enferm \\
\hline Sindicali (no) & Hijos (si) & Sexo (mascu) & Escolar (Prof) & Sala (5+) & Edad (31a50) & Enferm \\
\hline Sindicali (no) & Hijos (si) & Sexo (mascu) & Escolar (Prof) & Sala (5+) & Edad (51+) & Calam \\
\hline Sindicali (no) & Hijos (no) & Contrato (fijo) & Casado (si) & Enfermedad & & \\
\hline Sindicali (no) & Hijos (no) & Contrato (fijo) & Casado (no) & Calamidad & & \\
\hline Sindicali (no) & Hijos (no) & Contrato (indef) & Enfermedad & & & \\
\hline
\end{tabular}




\section{DISCUSIÓN}

Un análisis de los resultados permite ver, que según los datos de la Tabla 4, la metodología propuesta tiene una efectividad del $94.72 \%$. Igualmente, se observa que la medida de concordancia denotada por el índice Kappa presenta un nivel bastante alto, aspecto que determina la solidez de los resultados. Respecto a la Tabla 5 se encuentra que el porcentaje de resultados denominados positivos verdaderos es bastante alto 0.953 y 0.935 , para cada uno de los dos estados de la variable dependiente enfermedad y calamidad respectivamente. En contraste, el porcentaje de falsos positivos es bastante bajo 0.065 y 0.047 para cada uno de los dos estados de la variable dependiente. Por su parte en la matriz de confusión (Tabla 6) se ilustran los valores absolutos de aciertos y errores para cada uno de los dos estados de la variable dependiente, respecto al estado denominado enfermedad, 265 instancias fueron clasificados correctamente y 13 incorrectamente. En una forma análoga, para el otro estado de la variable dependiente (calamidad) fueron clasificadas 130 instancias de forma correcta y nueve de forma incorrecta.

En una forma análoga, la Figura 1 ilustra las relaciones existes entre las variables independientes y la variable dependiente. Esta relación, permite definir los diferentes valores de las causas conducentes a un estado específico de la variable dependiente. Este aspecto permite establecer las razones que pueden llevar a un ausentismo por enfermedad o calamidad. Se resalta que en cada nodo terminal (hoja) el número de la izquierda representa las instancias que cumplen esos criterios y el número de la derecha (si lo hay) representa las instancias clasificadas incorrectamente. Un análisis más detallado de las relaciones de esta figura es ilustrado en la Tabla 8. En esta tabla se observa que los ausentismos por enfermedad solo se producen en personas no sindicalizadas. Igualmente, las causas más influyentes en los ausentismos por enfermedad son en orden de importancia: Sindicalizado, hijos, sexo, escolaridad, la antigüedad, salario, turno y edad. Por otro lado, las causas del ausentismo por calamidad son en orden de importancia: Sindicalizado, hijos, sexo, contrato, casado, escolaridad, salario y edad.

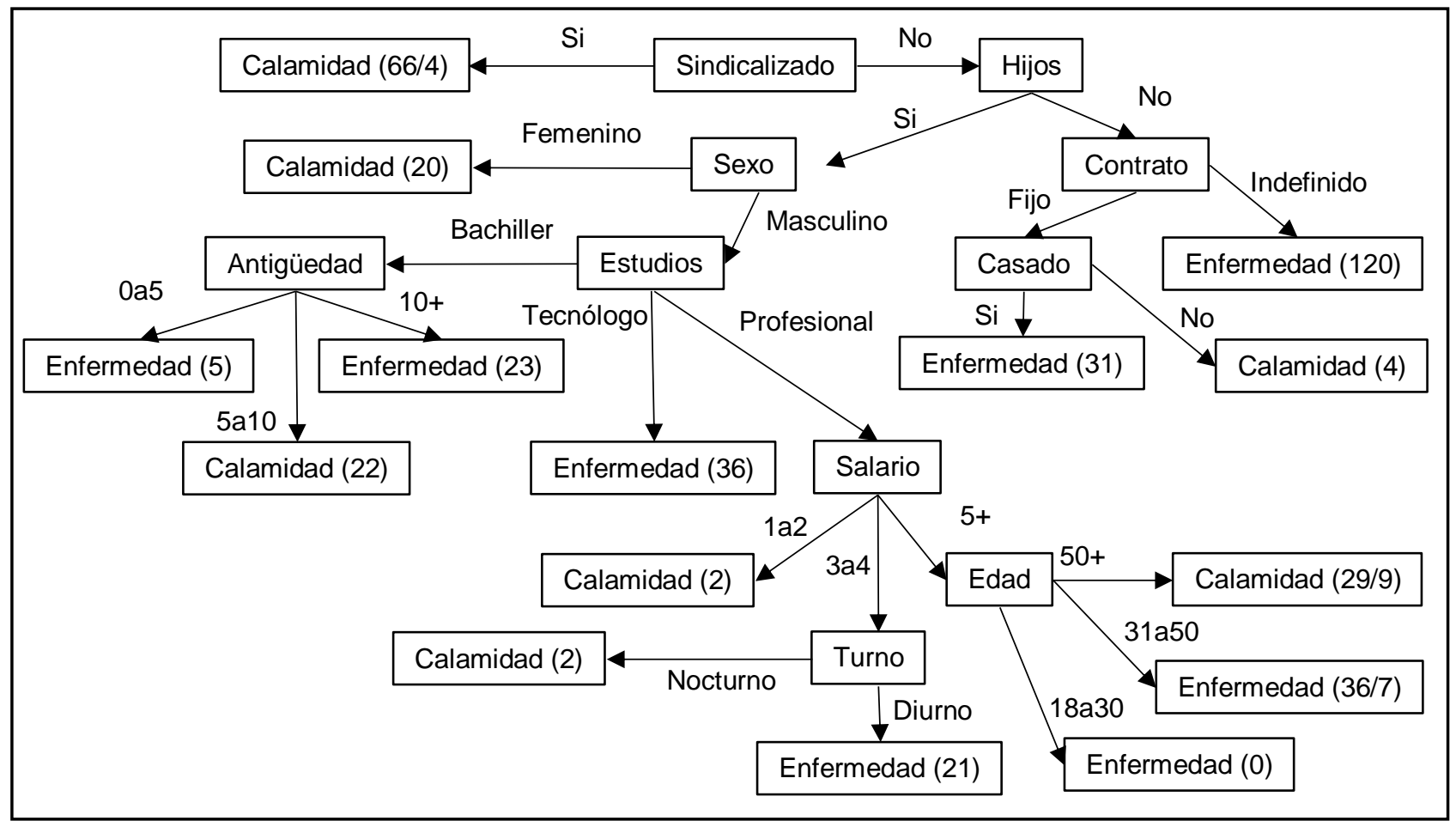

Fig.1: Árbol de clasificación

Desde otro punto de vista, al comparar los resultados obtenidos con las referencias revisadas, se observa que los análisis de ausentismo por medio de técnicas de inteligencia artificial y específicamente minería de datos, son escasos. Se encuentran algunos trabajos relacionados indirectamente (Darvishi et al., 2017; Pucheril et al., 2019) y un trabajo relacionado directamente (Thales y Costa, 2020). En este último trabajo, se logra una efectividad del $78 \%$ en la predicción de este problema. No obstante, estos autores, se enfocan en el estudio del ausentismo por enfermedad en trabajadores del servicio de seguridad, donde se analizan características como: lugar de trabajo, antigüedad, nivel en la institución, estado (activo, retirado), sexo, número de años de servicios y días de licencia. Esto autores, encuentra que las características más influyentes son: lugar de trabajo, sexo, edad, años de servicios e incapacidades. Si bien, se analizan características diferentes (variables independientes), a las estudiadas en este artículo, existen algunas coincidencias en relación con estas variables: Sexo, edad y antigüedad. No obstante, es importante resaltar que la selección de variables analizadas, pueden estar relacionadas con el tipo de población objeto de estudio. 
Como se expresó en el párrafo anterior, algunas de las variables principales (sexo, edad y antigüedad) identificadas en Thales y Costa (2020) coinciden con las encontradas en esta investigación. No obstante, la Tabla 8 muestra otras variables influyentes diferentes como: sindicalizado, hijos, contrato y escolaridad. Además, los resultados de este artículo, muestran que el ausentismo por enfermedad principalmente se da en hombres que no está sindicalizados, con hijos, bachilleres con muy poco o demasiado tiempo en la empresa. Así mismo, esta incapacidad se puede dar en hombres no sindicalizados entre los 18 y 50 años, con hijos, un nivel de formación profesional, y un sueldo superior a los 3 salarios mínimos.

Así mismo, este tipo de ausentismos se puede encontrar en personas no sindicalizadas sin hijos y con contratos a término fijo. En un sentido análogo, se puede decir que el ausentismo por calamidad se da en las personas sindicalizadas. En el caso de las personas no sindicalizadas, este ausentismo se da en hombres con hijos, bachilleres con una antigüedad entre 5 y 10 años ó un sueldo entre 1 a 2 salarios. También, este ausentismo puede ser encontrado en hombres con hijos, bachilleres, con trabajo nocturno y una remuneración entre 3 y 4 salarios mínimos o en hombres mayores de 50 años, con hijos bachilleres y un sueldo mayor a los 5 salarios mínimos.

Por otra parte, este trabajo difiere de los estudios realizados por (Darvishi et al., 2017), quienes desarrollaron un modelo basado en la predicción de enfermedades que pueden llevar a un ausentismo laboral. Sin embargo, la propuesta de estos autores no permite explicar los ausentismos por calamidad, los cuales no están asociados a enfermedades. Además, es importante resaltar que el enfoque de este trabajo no está en determinar las enfermedades que pueden llevar a un ausentismo, sino las causas de diferente índole que pueden producir el mismo. Es importante resaltar que las causas del ausentismo laboral pueden diferir según las bases de datos empleadas.

Como se ilustra en esta sección solo tres causas de ausentismo encontradas en esta investigación (sexo, edad y antigüedad) coinciden con la literatura internacional, sin embargo, las particularidades de cada contexto, país y región pueden explicar estos aspectos. Siendo importante resaltar que esta investigación permite diseñar una metodología bajo software libre la cual puede ser replicable en cualquier empresa o conglomerado de ellas (sin emplear bases de datos extensas o internacionales dado que los algoritmos empleados son confiables con pocos datos), con el fin de que las empresas obtengan sus propios resultados y desarrollen sus propios análisis.

Los anteriores aspectos, permiten trazar políticas efectivas, para prevenir el ausentismo en las empresas, tanto por enfermedad como por calamidad. Este aspecto traerá grandes beneficios económicos, sociales y laborales para la organización que adopte esta metodología. Aunque es importante resaltar que las variables que afectan el ausentismo pueden variar según el sector y contexto en el que se encuentre ubicada la empresa.

\section{CONCLUSIONES}

En esta investigación se logra con una efectividad superior al $94.72 \%$ identificar las principales causas que pueden intervenir en el ausentismo por calamidad o enfermedad en una empresa. Se identifican las siguientes variables como principales causas de este fenómeno: Sindicalizado, hijos, sexo, contrato, estudios, casado y antigüedad. Igualmente, el desarrollo metodológico llevado acabo, define una forma objetiva para resolver un problema tradicionalmente analizado de forma subjetiva el cual puede ser replicable si se siguen los pasos estructurados en la metodología propuesta.

\section{AGRADECIMIENTOS}

Se agradece la colaboración a la Universidad Nacional de Colombia y en especial al Departamento de Ingeniería Industrial de la Sede Manizales.

\section{REFERENCIAS}

Askun-Celik, D. y Unler-Oz, E., The Effects of Emotional Dissonance and Quality of Work Life Perceptions on Absenteeism and Turnover Intentions Among Turkish Call Center Employees, doi:10.1016/j.sbspro.2011.10.491, Procedia - Social and Behavioral Sciences, 30, $2515-2519$ (2011)

Ben-David, T., Rackes, A. y Waring, M., Alternative Ventilation Strategies in U.S. Offices: Saving Energy While Enhancing Work Performance, Reducing Absenteeism, and Considering Outdoor Pollutant Exposure Tradeoffs, http://dx.doi.org/10.1016/j.buildenv.2017.02.004, Building and Environment, 116, 140 -157 (2017)

Bennedsen, M., Tsoutsoura, M., y Wolfenzon, D., Drivers of Effort: Evidence from Employee Absenteeism, https://doi.org/10.1016/j.jfineco.2018.12.001, Journal of Financial Economics, 133, 658-684 (2019) 
Castrillón, O., Sarache, W. y Ruiz, H., Predicción del Rendimiento Académico por Medio de Técnicas de Inteligencia Artificial, http://dx.doi.org/10.4067/S0718-50062020000100093, Formación Universitaria, 13(1), 93 -102 (2020)

Castrillón, O., Sarache, W. y Castaño, E., Sistema Bayesiano para la Predicción de la Diabetes, http://dx.doi.org/10.4067/S0718-07642017000600017, Información Tecnológica, 28 (6), 161-168 (2017)

Cucchiella, F., Gastaldi, M. y Ranieri, L., Managing Absenteeism in the Workplace: the Case of an Italian Multiutility Company, doi: 10.1016/j.sbspro.2014.09.131, Procedia - Social and Behavioral Sciences, 150, 1157 - 1166 (2014)

Cuevas, Y-S., García, T. y Villa Marilyn, Caracterización del Ausentismo Laboral en un Centro Médico de I Nivel, Tesis Especialista en Salud Ocupacional, https://repository.urosario.edu.co/bitstream/handle/10336/2834/22647541 2012.pdf;jsessionid=F9137B363B53E6679F6E511E54939405?sequence=1, Colegio Mayor Nuestra Señora del Rosario, $1-45(2011)$

Darvishi, E., Khotanlou, H. y Otros 3 autores, Prediction Effects of Personal, Psychosocial, and Occupational Risk Factors on Low Back Pain Severity Using Artificial Neural Networks Approach in Industrial Workers, http://dx.doi.org/10.1016/j.jmpt.2017.03.012, Journal of Manipulative and Physiological, 40 (7), $486-493$ (2017)

Enns, V., Currie, S. y Wang, J., Professional Autonomy and Work Setting as Contributing Factors to Depression and Absenteeism in Canadian Nurses, http://dx.doi.org/10.1016/j.outlook.2014.12.014, Nurse Out look, 63, 269 - 277 (2015)

Halbesleben J., Whitman, M. y Crawford W., A Dialectical Theory of the Decision to Go to Work: Bringing Together Absenteeism and Presenteeism, http://dx.doi.org/10.1016/j.hrmr.2013.09.001, Human Resource Management Review, 24, 177-192, (2014)

Heinemann, I., Minh, T-D. y Otros 2 autores, Explorative Evaluation of the Impact of Severe Premenstrual Disorders on Work Absenteeism and Productivity, doi:10.1016/j.whi.2009.09.005, Women's Health Issues, 20, 58-65 (2010)

Hernández-Martínez, J., Varona-Uribe, M. y Hernández, G., Prevalencia de Factores Asociados a la Enfermedad Cardiovascular y su Relación con el Ausentismo Laboral de los Trabajadores de una Entidad Oficial, Rev Colomb Cardiol, $27(2), 109-116(2020)$

Johnston, D.A., Harvey, S.B. y Otros 4 autores, The Relationship Between Depression Symptoms, Absenteeism and Presenteeism, https://doi.org/10.1016/j.jad.2019.06.041, Journal of Affective Disorders, 256, 536-540 (2019)

Karatepe, O., Rezapouraghdam, H. y Hassannia, R., Job Insecurity, Work Engagement and their Effects on Hotel Employees' Non green and Nonattendance Behaviors, https://doi.org/10.1016/j.jjhm.2020.102472, International Journal of Hospitality Management, 87, 102472 (2020)

Murti, M., Otterstatter, M. y Otros 19 autores, Measuring the Impact of a Mandatory Province-Wide Vaccinate-or-Mask Policy on Healthcare Worker Absenteeism in British Columbia, Canada, https://doi.org/10.1016/j.vaccine.2019.06.007, Vaccine, 37, 4008-4014 (2019a)

Murti, M., Otterstatter, M. y Otros 20 autores, Measuring the Impact of Influenza Vaccination on Healthcare Worker Absenteeism in the Context of a Province-Wide Mandatory Vaccinate or Mask Policy, https://doi.org/10.1016/j.vaccine.2019.06.014, Vaccine, 37, 4001-4007 (2019b)

Pucheril, D.T., Chen, X. y Otros 5 autores, Fewer Days of Workplace Absenteeism with Robotic Radical Prostatectomy Compared to Open Radical Prostatectomy, DOI: 10.1016/S1569-9056(19)31582-9, Eur Urol Suppl, 18(1), e2189 (2019)

Restrepo, C. y Salgado E, Types of Contracts and Worker Absenteeism in Colombia, doi:10.1016/j.jbusres.2012.04.006, Journal of Business Research, 66, 401-408 (2013)

Saidane, O., Mahmoud, I. y Otros 4 Autores, Factors Leading to Work Absenteeism in Tunisian Ankylosing Spondylitis Patients, http://dx.doi.org/10.1016/j.ejr.2017.06.009, The Egyptian Rheumatologist, 40, 183-185 (2018)

Strömberg, C., Aboagye, E. y Otros 3 autores, Estimating the Effect and Economic Impact of Absenteeism, Presenteeism, and Work Environment - Related Problems on Reductions in Productivity from a Managerial Perspective, http://dx.doi.org/10.1016/j.jval.2017.05.008, Value in Health, 20, 1058 - 1064 (2017)

Thales E., Costa E., Evaluating Deep Models for Absenteeism Prediction of Public Security Agents, https://doi.org/10.1016/j.asoc.2020.106236, Applied Soft Computing Journal, 91 , 106236 (2020)

Valencia, M., Correa, J. y Díaz, F., Métodos Estadísticos Clásicos y Bayesianos para el Pronóstico de Demanda. Un Análisis Comparativo, https://doi.org/10.15446/rev.fac.cienc.v4n1.49775, Revista Facultad de Ciencias Universidad Nacional de Colombia, 4(1), 52 -67 (2015)

Verstappen, S., Rheumatoid Arthritis and Work: The impact of Rheumatoid Arthritis on Absenteeism and Presenteeism, http://dx.doi.org/10.1016/j.berh.2015.06.001, Best Practice \& Research Clinical Rheumatology, 29, 495e511 (2015)

Witten, I., Frank, E. y otros dos autores, Data Mining Practical Machine Learning Tools and Techniques, Morgan and Kaufman publication (Elsevier), ISBN-13: 978-0128042915, Cambrige, USA (2017) 